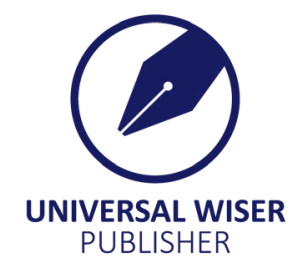

\title{
On the Non-Uniqueness of the Sets Computing a Partially Symmetric Rank at Most Three
}

\section{Edoardo Ballico}

Department of Mathematics, University of Trento, 38123 Povo (TN), Italy

Email: ballico@science.unitn.it

Abstract: We describe all partially symmetric tensors which have rank two in more than one way and gives many examples, perhaps all, for rank three partially symmetric tensors.

Keywords: $X$-rank, Segre-Veronese variety, partially symmetric rank

\section{Introduction}

Let $Y=\mathbb{P}^{n_{1}} \times \cdots \times \mathbb{P}^{n_{k}}$ be a multiprojective space. For all $\left(d_{1}, \ldots, d_{k}\right) \in(\mathbb{N} \backslash\{0\})^{k}$ let $v_{\left(d_{1}, \ldots, d_{k}\right)}: Y \rightarrow \mathbb{P}^{r}, r=-1+$ $\prod_{i=1}^{k}\left(\begin{array}{c}n_{i}+d_{i} \\ n_{i}\end{array}\right)$, denote the Segre-Veronese embedding of $Y$, i.e. the embedding of $Y$ by the complete linear system $\mid \mathcal{O}_{Y}\left(d_{1}, \ldots\right.$, $\left.d_{k}\right)$. The case $k=1$ is just the Veronese embedding of the projective space $\mathbb{P}^{n_{1}}$. The case $d_{i}=1$ for all $i$ corresponds to the Segre embedding of $Y$. Set $X:=v_{d_{1}, \ldots, d_{k}}(Y)$. We recall that the elements of $H^{0}\left(\mathcal{O}_{Y}(1, \ldots, 1)\right)^{\vee}$ correspond to the partially symmetric tensors of format $\left(\begin{array}{c}n_{1}+d_{1} \\ n_{1}\end{array}\right) \times \cdots \times\left(\begin{array}{c}n_{k}+d_{k} \\ n_{k}\end{array}\right)$ and hence the elements of $\mathbb{P}^{r}$ correspond to partially symmetric tensors of that format, up to a non-zero multiplicative constant. Fix $q \in \mathbb{P}^{r}$. The $X$-rank $r_{X}(q)$ of $q$ (or the partially symmetric rank of any non-zero tensor with $q$ as its equivalence class) is the minimal cardinality of a finite set $S \subset Y$ such that $q \in\left\langle v_{d_{1}, \ldots, d_{k}}(S)\right\rangle$, where \langle\rangle denote the linear span. The solution set $\mathcal{S}(X, q)$ is the set of all $S \subset Y$ such that $\# S=r_{X}(q)$ and $q \in\left\langle v_{d_{1}, \ldots, d_{k}}(S)\right\rangle$. Obviously $\mathcal{S}(X, q)=\emptyset$. We recall that $q \in \mathbb{P}^{r}$ is said to be a concise tensor or a concise partially symmetric tensor if there is no multiprojective space $Y^{\prime} \subsetneq Y$ such that $q \in\left\langle v_{d_{1}, \ldots, d_{k}}\left(Y^{\prime}\right)\right\rangle$.

In this note we prove the following result.

Theorem 1.1 Fix an integer $k \geq 1$ and positive integers $n_{i}, d_{i}, 1 \leq i \leq k$, such that $\left(d_{1}, \ldots, d_{k}\right) \neq(1, \ldots, 1)$. There is a concise tensor $q$ with $r_{X}(q)=2$ and $\# \mathcal{S}(X, q) \neq 1$ if and only if either $k=1, n_{1}=1, d_{1}=2$ and $\mathcal{S}(X, q)$ is $\mathbb{P}^{1}$ minus two points or $k=2, n_{1}=n_{2}=1,\left(d_{1}, d_{2}\right) \in\{(2,1),(1,2)\}$ and $q, \mathcal{S}(X, q)$ are as in Example 3.2.

We discuss several examples with $r_{X}(q)=3$ and $\# \mathcal{S}(X, q)>1$ and we wonder if they are the only ones. In particular we described all cases with $\# \mathcal{S}(X, q)>1$ when $r_{X}(q)=3$ and $q \in \tau(X)$ (Proposition 4.1). We always assume $d_{i} \geq 2$ for at least one integer $i$, because the case of the Segre variety is done in [4].

Question 1.2 Let $X \subset \mathbb{P}^{r}$ be an integral and non-degenerate variety. What is the maximal integer $\alpha_{X}>0$ (resp. $\alpha_{X}^{\prime}$ ) such that for each set $A \subset X$ with $\# A \leq \alpha_{X}-1$ and $A \in \mathcal{S}(X, q)$ for some $q \in \mathbb{P}^{r}$, we have $r_{X}(q)=\# A+1$ for a general $o \in X$ and a general $q^{\prime} \in\langle\{o, q\}\rangle$ (resp. all $\left.q^{\prime} \in\left\langle\left\{o, q^{\prime}\right\}\right\rangle \backslash\left\{o, q^{\prime}\right\}\right)$ ?

Obviously the integer $\alpha_{X}$ in Question 1.2 is at most the generic $X$-rank $r_{X, \text { gen }}$ of $\mathbb{P}^{r}$, i.e. the minimal integer $t$ such that $\sigma_{t}(X)=\mathbb{P}^{r}$, where $\sigma_{t}(X)$ denote the $t$-secant variety of $X^{[12-13]}$. In very special cases $r_{X, \text { gen }}=\alpha_{X}$. For instance this is true if $X$ is a rational normal curve by Sylvester's theorem ${ }^{[9,12-13]}$.

We work over an algebraically closed field of characteristic 0 .

\section{Notation and preliminary remarks}

Let $Y=\mathbb{P}^{n_{1}} \times \cdots \times \mathbb{P}^{n_{k}}, k \geq 1, n_{i} \geq 1$ for all $i$, be any multiprojective space. For any $\mathrm{i} \in(1, \ldots, k\}$ let $\pi_{i}: Y \rightarrow \mathbb{P}^{n_{i}}$ denote the projection onto the $i$-th factor of $Y$. If $k \geq 2$ set $Y_{i}:=\Pi_{h \neq i} \mathbb{P}^{n_{h}}$ and let $\eta_{i}: Y \rightarrow Y_{i}$ the morphisms which forget the $i$-th coordinate of any $p=\left(p_{1}, \ldots, p_{k}\right) \in Y$. Let $\varepsilon_{i}$ (resp. $\hat{\varepsilon}_{i}$ denote the element $\left(a_{1}, \ldots, a_{k}\right) \in \mathbb{N}^{k}$ with $a_{h}=1$ for all $h \neq i$ and $a_{i}=$ $0\left(\right.$ resp $a_{i}=0$ and $a_{h}=1$ for all $\left.h \neq i\right)$. Thus $\hat{\varepsilon}_{i}+\varepsilon=(1, \ldots, 1)$.

Remark 2.1 Fix $Y=\mathbb{P}^{n_{1}} \times \cdots \times \mathbb{P}^{n_{k}}$ and $\left(d_{1}, \ldots, d_{k}\right) \in(\mathbb{N} \backslash\{0\})^{k}$. Let $v_{d_{1}, \ldots, d_{k}}: Y \rightarrow \mathbb{P}^{r}, r=-1+\prod_{i=1}^{k}\left(\begin{array}{c}n_{i}+d_{i} \\ n_{i}\end{array}\right)$, be the Segre- 
Veronese embedding of $Y$ with multidegree $\left(d_{1}, \ldots, d_{k}\right)$. Let $Y^{\prime} \subseteq \mathrm{Y}$ be a multiprojective subspace. Obviously $v_{d_{1}, \ldots, d_{k} \mid \gamma^{\prime}}$ is the Segre-Veronese embedding of $Y^{\prime}$ with multidegree $\left(d_{1}, \ldots, d_{k}\right)$. Fix $q \in\left\langle v_{d_{1}, \ldots, d_{k}}\left(Y^{\prime}\right)\right\rangle$. It is known that $r_{v_{d_{1}, \ldots, d_{k}}(Y)}(q)$ $=r_{v_{d_{1}, \ldots, d_{k}}\left(Y^{\prime}\right)}(q)$ and that $\mathcal{S}\left(v_{d_{1}, \ldots, d_{k}}(Y), q\right)=\mathcal{S}\left(v_{d_{1}, \ldots, d_{k}}\left(Y^{\prime}\right), q\right)$. We will call Autarky or concision this property. For any finite set $A \subset Y$ the multiprojective space $\prod_{i=1}^{k}\left\langle\pi_{i}(A)\right\rangle$ is the minimal multipro jective subspace of $Y$ containing $A$. Thus Autarky means that for any $q^{\prime} \in \mathbb{P}^{r}$ knowing one solution $S^{\prime} \in \mathcal{S}\left(v_{d_{1}, \ldots, d_{k}}(Y), q^{\prime}\right)$ we reconstruct the minimal multiprojective space $Y^{\prime}$ $\subseteq Y$ such that $q^{\prime} \in\left\langle v_{d_{1}, \ldots, d_{k}}\left(Y^{\prime}\right)\right\rangle$. Note that $Y^{\prime}$ is uniquely determined by $q^{\prime}$.

Let $D \subset Y$ be an effective divisor. For any line bundle $\mathcal{L}$ on $Y$ and any finite set $S \subset Y$ there is an exact sequence

$$
0 \rightarrow \mathcal{I}_{S \backslash S \cap D} \otimes \mathcal{L}(-D) \rightarrow \mathcal{I}_{S} \otimes \mathcal{L} \rightarrow \mathcal{L}_{S \cap D, D} \rightarrow 0
$$

\section{Proof of theorem 1.1}

In this section we prove Theorem 1.1. By Autarky we have $n_{i}=1$ for all $i$.

Remark 3.1 Assume $k=1$ and hence $Y=\mathbb{P}^{1}$. Fix $q \in \mathbb{P}^{r}, r=d_{1}$, with $r_{X}(q)=2$. The case $d_{1} \geq 3$ is excluded by Sylvester's theorem ${ }^{[9,12]}(1.36,1.40)$. Now assume $d_{1}=2$ and hence $r=2$ and $X \subset \mathbb{P}^{2}$ and $r_{X}(q)=2$ if and only if $q \in \mathbb{P}^{r}$ $\backslash X$. The constructible set $\mathcal{S}(X, q)$ is isomorphic to the pencil of lines $L \subset \mathbb{P}^{2}$ containing $q$, minus the tangent lines to $X$ containing $q$. Since we are not in characteristic 2, there are exactly 2 lines passing through $q$ and tangent to $X$.

Example 3.2 Take $n_{1}=n_{2}=1$ and $\left(d_{1}, d_{2}\right) \in\{(1,2),(2,1)\}$. Just to fix the notation we assume $d_{1}=2$ and $d_{2}=1$. We have $\sigma_{2}(X)=\mathbb{P}^{5}$. It is well-known that in this case $X$ is an OADP, i.e. $\# \mathcal{S}(X, q)=1$ for a general $q \in \mathbb{P}^{r[8,10,17]}$; in the terminology of [8, Proposition 2.3] $X$ is the scroll $S(2,2))$. We will prove that all $q \in \mathbb{P}^{5} \backslash X$ have $r_{X}(q)=2$, that $\# \mathcal{S}(X, q)=$ 1 if $q \in \mathbb{P}^{5} \backslash \tau(X)$ and that there are two types of $q \in \tau(X) \backslash X$, one with $\operatorname{dim} \mathcal{S}(X, q)=1$ and one with $\operatorname{dim} \mathcal{S}(Y, q)=3$.

Take $L \in\left|\mathcal{O}_{Y}(1,0)\right|$. Note that $D:=v_{2,1}(L)$ is a smooth conic and $\langle D\rangle \nsubseteq X$. Hence each $q \in\langle D\rangle \backslash X \cap\langle D\rangle$ has $r_{X}(q)=$ 2 and $\mathcal{S}(X, q)$ is infinite. More precisely there is a 1-dimensional family of $\mathcal{S}(X, q)$ formed by the solutions spanning a line contained in $\langle D\rangle$.

Claim 1: Assume $q \in\langle D\rangle \backslash X \cap\langle D\rangle$. Every $A \in \mathcal{S}(X, q)$ is contained in $D$ and $\mathcal{S}(X, q)$ is isomorphic to $\mathbb{P}^{1}$ minus 2 points. Moreover $A \cap A^{\prime}=\varnothing$ for all $A, A^{\prime} \in \mathcal{S}(X, q)$ such that $A \neq A^{\prime}$.

Proof of Claim 1: The set of all $E \subset L$ such that $\# E=2$ and $q \in\left\langle v_{(2,1)}(E)\right\rangle$ is isomorphic to the set of all lines $T \subset\left\langle v_{(2,1)}(D)\right\rangle$ containing $q$ and not tangent to $D$ and hence is isomorphic to $\mathbb{P}^{1}$ minus 2 points. Note that any two such different elements are disjoint. Fix $A \in \mathcal{S}(X, q)$. There is $B \subset D$ such that $B \in \mathcal{S}(X, q)$ and $B \cap A=\varnothing$, because there is a line $T \subset\left\langle v_{(2,1)}(D)\right\rangle$ containing $q$, not tangent to $D$ and with $T \subset v_{(2,1)}(A)=\varnothing$. Set $S:=A \cup B$ and assume $A \nsubseteq D$, i.e. $S \backslash S \cap$ $D=\varnothing$. Since $S \nsubseteq D,\left[2\right.$, Lemma 5.1] gives $h^{1}\left(\mathcal{I}_{S \backslash S \cap D}(1,1)\right)>0$. Since $B \subset D, \#(S \backslash S \cap D) \leq 2$. Thus the very ampleness of $\mathcal{O}_{Y}(1,1)$ gives $h^{1}\left(\mathcal{I}_{S \backslash S \cap D}(1,1)\right)=0$, a contradiction.

Note that any $q$ as in Claim 1 is an element of $\tau(X) \backslash X$. All other elements of $\tau(X) \backslash X$ are obtained in the following way. Let $v \subset Y$ be a connected degree 2 zero-dimensional scheme contained neither in some $\mathrm{L} \in\left|\mathcal{O}_{Y}\left(\varepsilon_{1}\right)\right|$ nor in some $R$ $\in\left|\mathcal{O}_{Y}\left(\varepsilon_{2}\right)\right|$ (because $v_{2,1}(R) \subset X$ and so $\left\langle v_{2,1}(v) \subset X\right.$ if $v \subset R$ ). There is a smooth $C \in\left|\mathcal{I}_{v}(1,1)\right| . \operatorname{Since} \operatorname{dim}\left\langle v_{2,1}(C)\right\rangle=3$ and $v_{2,1}(C)$ is a rational normal curve of $\left\langle v_{2,1}(C)\right\rangle$, Sylvester's theorem gives $r_{X}(q)=3$ and $\operatorname{dim} \mathcal{S}\left(v_{2,1}(C)\right)=2$ for all $q$ $\in\left\langle v_{2,1}(v)\right\rangle \backslash v_{2,1}\left(v_{\text {red }}\right)$. Since there are $\infty^{1} C \in\left|\mathcal{I}_{v}(1,1)\right|$ and any two of them meet only along $v\left(\right.$ because $\mathcal{O}_{Y}(1,1) \cdot \mathcal{O}_{Y}(1,1)$ $=2)$, we get $\operatorname{dim} \mathcal{S}(Y, q)=3$.

Claim 2: Fix $a \in \sigma_{2}(X) \backslash \tau(X)$. We have $r_{X}(a)=2$ and $\# \mathcal{S}(X, a)=1$.

Proof of Claim 2: Since $X$ is smooth and $a \in \sigma_{2}(X) \backslash \tau(X), r_{X}(a)=2$. Assume that $\mathcal{S}(Y, a)$ is not a singleton and take $E, F \in \mathcal{S}(X, a)$ such that $E \neq F$. Set $G:=E \cup F$. Since any two different lines either are disjoint or meets at one point and $a \in\left\langle v_{2,1}(E)\right\rangle \cap\left\langle v_{2,1}(F)\right\rangle$, we have $E \cap F=0$. Hence $h^{1}\left(\mathcal{I}_{G}(1,1)\right)>0^{[1]}($ Lemma 1$)$. Thus any $C \in\left|\mathcal{O}_{Y}(1,1)\right|$ containing 3 points of $G$ contains the fourth one. Thus there is $C \in\left|\mathcal{I}_{G}(1,1)\right|$. Since $a \notin \tau(X)$, we saw that neither $E$ nor $F$ are contained in a ruling of $Y$. Thus $C$ is smooth. By assumption $a / \tau\left(v_{2,1}(C)\right)$. Hence $r_{v_{2,1}(C)}(a)=2$ and $E, F \in \mathcal{S}\left(v_{2,1}(C), a\right)$, contradicting ${ }^{[12]}$ (Theorem 1.40).

Proof of Theorem 1.1: Remark 3.1 describes the case $k=1$. From now on we assume $k \geq 2$.

See Example 3.2 for the case $k=2, n_{1}=n_{2}=1$ and $\left(d_{1}, d_{2}\right) \in\{(1,2),(2,1)\}$.

(b) Assume $k=2, d_{1}=d_{2}=2$. Take $H \in\left|\mathcal{O}_{Y}(2,0)\right|$ containing $A$. Either $S \subset H$ or $h^{1}\left(\mathcal{I}_{B \backslash B \cap H}(0,2)\right)>0^{[2-3]}($ Lemma 5.1 or Lemma 2.4).

(b1) First assume $S \subset H$. Since q is concise, there is no $M \in\left|\mathcal{O}_{Y}(1,0)\right|$ containing $S$. Since $S$ is a finite set, we get that $H \neq 2 M$ for any $M \in\left|\mathcal{O}_{Y}(1,0)\right|$. Thus $H=H \cup H^{\prime \prime}$ with $H^{\prime}, H^{\prime \prime} \in\left|\mathcal{O}_{Y}(1,0)\right|$. With no loss of generality we may assume $\#(S$ $\left.\cap H^{\prime}\right) \geq \#\left(S \cap H^{\prime \prime}\right)$. By [2, Lemma 5.1] or [3, Lemma 2.4] we have $h^{1}\left(\mathcal{I}_{S \cap H^{\prime \prime}}(1,1)\right)>0$. Since $\#\left(S \cap H^{\prime \prime}\right) \leq 2$ and $\mathcal{O}_{Y}(1,2)$ 
is very ample, we get a contradiction.

(b2) Now assume $h^{1}\left(H, \mathcal{I}_{B \backslash B \cap H}(0,2)\right)>0$. Since $h^{1}\left(\mathbb{P}^{1}, \mathcal{I}_{Z}(2)\right)=0$ for any scheme $Z \subset \mathbb{P}^{1}$ with $\operatorname{deg}(Z) \leq 3$, we get \#(B $B \cap H)=2$ (i.e. $B \cap H=\varnothing$ and $\#\left(\pi_{1}(B)\right)=1$. Set $M:=\pi_{1}^{-1}\left(\pi_{1}(M)\right) \in\left|\mathcal{I}_{B}(1,0)\right|$. Since $\mathcal{O}_{Y}(1,2)$ is very ample, we have $h^{1}\left(\mathcal{I}_{A}(1\right.$, 1)) = 0. Since $S \backslash S \cap M \subseteq A$, [2, Lemma 5.1] or [3, Lemma 2.4] give $S \subset M$, contradicting the assumption that $q$ is concise.

(c) Assume $k=2, d_{1}=3$ and $d_{2}=1$. Fix $H \in\left|\mathcal{O}_{Y}(2,0)\right|$ containing $A$. By [2-3] either $S \subset H$ or $h^{1}\left(\mathcal{I}_{B \backslash B \cap H}(0,1)\right)>0$.

(c1) Assume $S \subset H$. Since $q$ is concise, there is no $M \in\left|\mathcal{O}_{Y}(1,0)\right|$ containing $S$. Since $S$ is a finite set, we get that $H$ $\neq 2 M$ with $M \in\left|\mathcal{O}_{Y}(1,0)\right|$. Thus $H=H^{\prime} \cup H^{\prime \prime}$ with $H^{\prime}, H^{\prime \prime} \in\left|\mathcal{O}_{Y}(1,0)\right|$ and $H^{\prime} \neq H^{\prime \prime}$. With no loss of generality we may assume $\#\left(S \cap H^{\prime}\right)>\#\left(S \cap H^{\prime \prime}\right)$. By [2, Lemma 5.1] or [3, Lemma 2.4] we have $h^{1}\left(\mathcal{I}_{S \cap H^{\prime \prime}}(2,1)\right)>0$. Since $\#\left(S \cap H^{\prime \prime}\right) \leq 2$ and $\mathcal{O}_{Y}(1,2)$ is very ample, we get a contradiction.

(c2) Assume $h^{1}\left(\mathcal{I}_{B \backslash B \cap M}(0,1)\right)>0$. Since $\mathcal{O}_{Y}(0,1)$ is spanned, we get $B \cap M=\varnothing$ and $\#\left(\pi_{1}(B)\right)=1$, contradicting concision.

(d) As in steps (b) and (c) we exclude all other cases with $k=2$. Among the cases with $k>2$ we immediately see that it is sufficient to exclude the case $k=3, d_{1}=2$ and $d_{2}=d_{3}=1$. Assume $k=3, d_{1}=2$ and $d_{2}=d_{3}=1$. Fix $H \in\left|\mathcal{O}_{Y}(1,0,0)\right|$ containing at least one point of $S$. By Autarky we have $S \not \subset H$ and hence $h^{1}\left(\mathcal{I}_{S \backslash S \cap H}(1,1,1)\right)>0$. Since $\#(S \backslash S \cap H) \leq 3$ and $v_{1,1,1}(Y)$ is cut out by quadrics, we get $\#(S \backslash S \cap H)=3$ (i.e. $\#(S \cap H)=1$ ) and the existence of $i \in(1,2,3\}$ such that $\#\left(\pi_{1}(S\right.$ $\backslash S \cap H))=1$. Take $M:=\pi_{1}^{-1}\left(\pi_{1}(S \backslash S \cap H)\right) \in\left|\mathcal{O}_{Y}\left(\varepsilon_{i}\right)\right|$. Since $\mathcal{O}_{Y}(2,1,1)\left(-\varepsilon_{i}\right)$ is spanned and $\#(S \cap H)=1$, [2, Lemma 5.1] or [3, Lemma 2.4] gives a contradiction.

\section{4. $r_{X}(q)=3, q \in t(X)$}

In this section we prove the following result.

Proposition 4.1 Fix $q \in \tau(X)$ with $2 \leq r_{X}(q) \leq 3, k \geq 2$. Then $Y=\mathbb{P}^{1} \times \mathbb{P}^{1},\left(d_{1}, d_{2}\right) \in\{(2,1),(1,2)\}$ and $q$ is as in Example 3.2.

By section 3 we may assume $r_{X}(q)=3$. Fix $A \in \mathcal{S}(X, q)$ and a degree 2 connected scheme $v \subset Y$ such that $q \in$ $\left\langle v_{d_{1}, \ldots, d_{k}}(v)\right\rangle$. Set $\{o\}:=v_{\text {red }}$. Since $\operatorname{deg}(v)=2$ and $Y$ is minimal among the multiprojective spaces containing $v$, we have $n_{i}=$ 1 for all $i$. With no loss of generality we may assume $d_{1} \geq d_{2} \geq \cdots \geq d_{k}>0$. By assumption $k \geq 2$ and $d_{1} \geq 2$. Set $E:=v \cup A$. We have $h^{1}\left(\mathcal{I}_{E}\left(d_{1}, \ldots, d_{k}\right)\right)>0^{[1]}$ (Lemma 1$)$.

(a) First assume $d_{1} \geq 3$. Take $T_{i} \in\left|\mathcal{O}_{Y}\left(\varepsilon_{i}\right)\right|, 1 \leq i \leq 3$, such that $T_{1} \cup T_{2} \cup T_{3} \supset$ A and call $T \subseteq T_{1} \cup T_{2} \cup T_{3}$ containing $A$. We have $v \nsubseteq T$, because $T$ is reduced and $\operatorname{deg}\left(\pi_{1}(v)\right)=2$. Thus $h^{1}\left(\mathcal{I}_{v}\left(0, d_{2}, \ldots, d_{k}\right)\right)>0^{[2-3]}($ Lemma 5.1 or Lemma 2.4), contradicting the assumptions $k \geq 2$ and $\operatorname{deg}\left(\pi_{2}(v)\right) \geq 2$.

(b) By step (a) from now on we assume $d_{1}=2$. Take $T \in\left|\mathcal{O}_{Y}\left(2 \varepsilon_{i}\right)\right|$ containing $v$. Note that $T=2 K$ with $\{K\}=\mid \mathcal{I}_{o}$ $\left(\varepsilon_{i}\right) \mid$. Either $h^{1}\left(\mathcal{I}_{A \backslash A \cap T}\left(0, d_{2}, \ldots, d_{k}\right)\right)>0$ or $A \subset T$ and hence $A \subset K$. The latter is impossible, because $K$ is a proper multiprojective subspace of $Y$. Thus $h^{1}\left(\mathcal{I}_{A \backslash A \cap T}\left(0, d_{2}, \ldots, d_{k}\right)\right)>0$. Since $Y$ is the minimal multiprojective space containing $A$ by Autarky, we have $\#\left(\pi_{1}(A)\right)>1$ for all $i$. Since $h^{1}\left(\mathcal{I}_{A \backslash A \cap T}\left(0, d_{2}, \ldots, d_{k}\right)\right)>0$, there are $a, b \in A \backslash A \cap T$ such that $\pi_{i}(a)=$ $\pi_{i}(b)$ for all $i>1$. Write $A=\{a, b, c\}$.

(b1) Assume $k \geq 3$. Take $\{M\}:=\left|\mathcal{I}_{a}\left(\varepsilon_{2}\right)\right|$ and $\left.M^{\prime}:=\mid \mathcal{I}_{c}\left(\varepsilon_{3}\right)\right) \mid$. Note that $A \subset M \cup M^{\prime}$. Since $h^{1}\left(\mathcal{I}_{v}\left(2 \varepsilon_{1}\right)\right)=0$, we get $v \subset$ $M \cup M^{\prime}$, i.e. $\pi_{2}(a)=\pi_{2}(o)$ and $\pi_{3}(c)=\pi_{3}(o)$. Using $\left.\mid \mathcal{I}_{c}\left(\varepsilon_{2}\right)\right) \mid$ and $\left|\mathcal{I}_{a}\left(\varepsilon_{3}\right)\right|$ we get $\pi_{2}(c)=\pi_{2}(o)$. Thus $\#\left(\pi_{2}(A)\right)=1$, contradicting the minimality of $Y$.

(b2) By $k=2$. If $d_{2}=1 q$ is as in Example 3.2. Assume $d_{2}=2$. Using $T^{\prime} \in\left|\mathcal{O}_{Y}\left(2 \varepsilon_{2}\right)\right|$ instead of $T$ as in the first part of step (b) we get the existence of $a^{\prime}, b^{\prime} \in A$ such that $a^{\prime} \neq b^{\prime}, \pi_{1}\left(a^{\prime}\right)=\pi_{1}\left(b^{\prime}\right)$ and $\pi_{2}\left(c^{\prime}\right)=\pi_{2}(o)$, where $\left\{c^{\prime}\right\}:=A \backslash\left(a^{\prime}, b^{\prime}\right\}$. Since $\#(A)=3$ and $\left(a^{\prime}, b^{\prime}\right\}=\{a, b\}$ we may assume $a^{\prime}=a$ and $b^{\prime}=c$. Thus $c^{\prime}=b$. Thus $\pi_{2}(b)=\pi_{2}(o)=\pi_{2}(c)$. Write $\{H\}:=\left|\mathcal{I}_{o}\left(\varepsilon_{2}\right)\right|$. We have $A \nsubseteq H$ by the minimality of $Y$. Since $\operatorname{Res}_{H}(E) \subseteq\{a, o\}$, we have $h^{1}\left(\mathcal{I}_{\operatorname{Res}_{H}(E)}(2,1)\right)=0$, contradicting [2-3]) and concluding the proof of Proposition 4.1.

\section{Other examples with $r_{X}(q)=3$}

Remark 5.1 Take $k=2, n_{1}=n_{2}=d_{2}=1$ and $d_{1}=2$. Thus $r=5$. The case $r_{X}(q)=2$ is done in Example 3.2. Since $X$ is not the Veronese surface, we have $\sigma_{2}(X)=\mathbb{P}^{5}$. Thus all $q \in \mathbb{P}^{5}$ with $r_{X}(q)=3$ are contained in $\tau(X) \backslash X$. These case is described in Example 3.2.

Remark 5.2 Take $k=2, n_{1}=n_{2}=1$ and $d_{1}=d_{2}=2$. Since $\operatorname{dim} \sigma_{3}(X)=7^{[14-16]}$, a general $q \in \sigma_{3}(X)$ has $\operatorname{dim} \mathcal{S}(Y, q)=1$. By [11, Ex. II.3.22, part (b)] every $q \in \mathbb{P}^{8}$ with $r_{X}(q)=3$ has $\operatorname{dim} \mathcal{S}(X, q) \geq 1$.

Remark 5.3 Take $k=3, n_{1}=n_{2}=n_{3}=1$ and 


$$
\left(d_{1}, d_{2}, d_{3}\right) \in\{(2,1,1),(1,2,1),(1,1,2)\} .
$$

Since $\operatorname{dim} \sigma_{3}(X)=10^{[5,7,15]}$, a general $q \in \sigma_{3}(X)$ has $\operatorname{dim} \mathcal{S}(X, q)=1$. [11, Ex. II.3.22, part (b)] every $q \in \mathbb{P}^{r}$ with $r_{X}(q)=$ 3 has $\operatorname{dim} \mathcal{S}(X, q) \geq 1$.

Remark 5.4 Take $k=4, n_{1}=n_{2}=n_{3}=n_{4}=1$ and $d_{1}=d_{2}=d_{3}=d_{4}=1$. Since $\operatorname{dim} \sigma_{3}(X)=13^{[6,15]}$, a general $q \in \mathbb{P}^{r}$ has $\operatorname{dim} \mathcal{S}(X, q)=1$. By [11, Ex. II.3.22, part (b)] every $q \in \mathbb{P}^{r}$ with $r_{X}(q)=3$ has $\operatorname{dim} \mathcal{S}(X, q) \geq 1$.

Remark 5.5 The case $k=1$, i.e. the case of Veronese embedding, is easy for points $q$ with $r_{X}(q)=3$. Note the existence of points of rank $>1$ implies $d_{1} \geq 2$. Since $r_{X}(q)=3$, concision gives $n_{1} \in\{1,2\}$. for each $q \in \mathbb{P}^{2} \backslash X(\mathcal{S}(Y, q)$ is the set of all lines of $\mathbb{P}^{2}$ through $q$ and not tangent to $X$. Sylvester's theorem ${ }^{[9,12]}$ says that there are no cases with $n_{1}=1$ and $d_{1} \neq 4$.

Claim 1: There is no $q$ with $r_{X}(q)=3$ and $\# \mathcal{S}(Y, q)>1$ with $n_{1}=2, q \notin\left\langle v_{d}(L)\right\rangle$ for any line $L \subset \mathbb{P}^{2}$ and $\mathrm{d}-1 \geq 4$.

Proof of Claim 1: Assume the existence of $q$ with $A, B \in \mathcal{S}(Y, q)$ and $A \neq B$. Set $S:=A \cup B$. Take a line $L \subset \mathbb{P}^{2}$ containing at least 2 points of $A$. We get $h^{1}\left(\mathcal{I}_{S \backslash S \cap L}(d-1)\right)>0^{[2-3]}$. Since $\#(S \backslash S \cap L) \leq 4$ and $d_{1}-1 \geq 3$, this is false.

Thus we get the following cases:

(1) $n_{1}=1, d_{1}=4, q$ sufficiently general in $\mathbb{P}^{4}$ with $\operatorname{dim} \mathcal{S}(Y, q)=1$; by [11, Ex. II.3.22] every $q \in \mathbb{P}^{4}$ with $r_{X}(q)=3$ has $\operatorname{dim} \mathcal{S}(X, q) \geq 1$

(2) $n_{2}=2, d_{1}=3, q$ sufficiently general in $\mathbb{P}^{5}$ with $\operatorname{dim} \mathcal{S}(Y, q)=3$; by [11, Ex. II.3.22] every $q \in \mathbb{P}^{5}$ with $r_{X}(q)=3$ has $\operatorname{dim} \mathcal{S}(X, q) \geq 3$.

Example 5.6 Take $n_{1}=2, n_{2}=1, d_{1}=1$ and $d_{2}=2$ (the same proof works for the case $\left.\left(n_{1}, n_{2}, d_{1}, d_{2}\right)=(1,2,2,1)\right)$. We have $r=8$. Since $\sigma_{3}(X)=\mathbb{P}^{8}$, we have $\operatorname{dim} \mathcal{S}(Y, q)=3$ for a general $q \in \mathbb{P}^{8}$. By [11, Ex. II.3.22] every $q \in \mathbb{P}^{8}$ with $r_{X}(q)=3$ has $\operatorname{dim} \mathcal{S}(X, q) \geq 1$.

Proposition 5.7 Set $d_{1}:=2$. Fix an integer $k \geq 2$ and take positive integers $d_{2}, \ldots, d_{k}$. Fix $n_{1} \in\{1,2\}$ and set $n_{i}:=1$ for all $i=2, \ldots, k$. Fix a line $L \subseteq \mathbb{P}^{n_{1}}$ and take $o_{1} \in \mathbb{P}^{n_{1}}$; if $n_{1}=2$ assume $o_{1} \notin L$. Fix $e_{i}, o_{i} \in \mathbb{P}^{1}, i=2, \ldots, k$ such that $e_{i} \neq o_{i}$ for all $i$. Set $Y:=\mathbb{P}^{n_{1}} \times\left(\mathbb{P}^{1}\right)^{k-1}, Y^{\prime}:=L \times\left\{e_{2}\right\} \times \cdots \times\left\{e_{k}\right\} \subset Y, o:=\left(o_{1}, \ldots, o_{k}\right), X:=v_{\left(d_{1}, \ldots, d_{k}\right)}(Y)$ and $X^{\prime}:=v_{\left(d_{1}, \ldots, d_{k}\right)}\left(Y^{\prime}\right)$. Fix $q^{\prime} \in\left\langle X^{\prime}\right\rangle \backslash X^{\prime}$ and take $q \in\left\langle\left\{q^{\prime}, v_{\left(d_{1}, \ldots, d_{k}\right)}(o)\right\}\right\rangle \backslash\left\langle\left\{q^{\prime}, v_{\left(d_{1}, \ldots, d_{k}\right)}(o)\right\}\right\rangle$. Then

(1) $Y$ (resp. $Y^{\prime}$ ) is the minimal multiprojective space containing $q$ (resp. $q^{\prime}$ ).

(2) $r_{X}\left(q^{\prime}\right)=r_{X}\left(q^{\prime}\right)=2, \mathcal{S}\left(X, q^{\prime}\right)=\mathcal{S}\left(X^{\prime}, q^{\prime}\right)$ is isomorphic to $\mathbb{P}^{1}$ minus 2 points.

(3) $2 \leq r_{X}(q) \leq 3$.

(4) Assume either $d_{2} \geq 2$ or $k \geq 3$. Then $r_{X}(q)=3$ and $\operatorname{dim} \mathcal{S}(X, q)>0$.

Proof. Since $\left\langle X^{\prime}\right\rangle \cong \mathbb{P}^{2}$ and $q^{\prime} \notin X^{\prime}, q^{\prime}$ is as in case (1) of Theorem 1.1 and hence $\mathcal{S}\left(X^{\prime}, q^{\prime}\right)$ is isomorphic to $\mathbb{P}^{1}$ minus 2 points. By Autarky $r_{X}\left(q^{\prime}\right)=r_{X^{\prime}}(q)$. By our choice of $o, Y$ is the minimal multiprojective space containing $Y^{\prime}$ and $o$. Since $q$ is in the linear span of $q^{\prime}$ and a point of $X, 1 \leq r_{X}(q) \leq 3$. Thus to prove part (3) it is sufficient to prove that $r_{X}(q)>1$. Assume $r_{X}(q)=1$, i.e. assume $q=v_{\left(d_{1}, \ldots, d_{k}\right)}(a)$ for some $a \in Y$. Since $q^{\prime} \in\left\langle\{q,\{o\}\rangle\right.$ and $r_{X}\left(q^{\prime}\right)=2$, we get $\{a, o\} \in \mathcal{S}\left(X, q^{\prime}\right)$, contradicting Autarky and the assumption $o_{2} \neq e_{2}$.

(a) Assume $d_{2} \geq 2$.

Assume $r_{X}(q)=2$ and take $B \in \mathcal{S}(X, q)$. Fix $A \in \mathcal{S}\left(X^{\prime}, q^{\prime}\right)$ and set $S:=A \cup B\{o\}$. Since $\left.q \in\left\langle v_{\left(d_{1}, \ldots, d_{k}\right)}(A)\right\rangle \cap_{\left(d_{1}, \ldots, d_{k}\right)}(B)\right\rangle$, $q \notin\left\langle v_{\left(d_{1}, \ldots, d_{k}\right)}\left(B^{\prime}\right)\right\rangle$ for any $B^{\prime} \subsetneq B$ and $B \nsubseteq A, h^{1}\left(\mathcal{I}_{S}\left(d_{1}, \ldots, d_{k}\right)\right)>0^{[1]}$ (Lemma 1). Let $M$ be the only element of $\left|\mathcal{O}_{Y}\left(\varepsilon_{2}\right)\right|$ such that $\pi_{2}(M)=\left\{e_{2}\right\}$. Consider the residual exact sequence of $M$ :

$$
0 \rightarrow \mathcal{I}_{S \backslash S \cap M}\left(d_{1}, d_{2}-1, \ldots, d_{k}\right) \rightarrow \mathcal{I}_{S}\left(d_{1}, \ldots, d_{k}\right) \rightarrow \mathcal{I}_{S \cap M, M}\left(d_{1}, \ldots, d_{k}\right) \rightarrow 0
$$

Since $S \not \nsubseteq M, h^{1}\left(\mathcal{I}_{S \backslash \cap \cap M}\left(d_{1}, d_{2}-1, \ldots, d_{k}\right)\right)>0^{[2-3]}$ (Lemma 5.1, Lemma 2.4). Since $\mathcal{O}_{Y}\left(d_{1}, d_{2}-1, \ldots, d_{k}\right)$ is very ample, $\#(S \backslash S \cap M) \geq 3$. Thus $\#(S \backslash S \cap M)=3$, i.e. $(A \cup\{o\}) \cap M=\varnothing$ and $S \backslash S \cap M=A \cup\{o\}$. Since $h^{1}\left(\mathcal{I}_{S \backslash S \cap M}\left(d_{1}, d_{2}-1, \ldots\right.\right.$, $\left.\left.d_{k}\right)\right)>0, v_{\left(d_{1}, \ldots, d_{k}\right)}(A \cup\{o\})$ is formed by 3 collinear points. Thus $v_{\left(d_{1}, \ldots, d_{k}\right)}(o) \in\left\langle X^{\prime}\right\rangle$.

Since $v_{\left(d_{1}, \ldots, d_{k}\right)}(o) \notin\left\langle X^{\prime}\right\rangle$, we get $\left.\left.r_{X^{\prime}}\left(v_{\left(d_{1}, \ldots, d_{k}\right)}\right)(o)\right)>r_{X}\left(v_{\left(d_{1}, \ldots, d_{k}\right)}\right)(o)\right)$, contradicting Autarky. Thus $r_{X}(q)=3$. Hence $\mathcal{S}(X$, $q) \supseteq\{\{o\} \cup A\}_{A \in \mathcal{S}\left(X^{\prime}, q\right)}$. Thus $\operatorname{dim} \mathcal{S}(X, q) \geq 1$.

(b) Assume $d_{2}=1$ and $k \geq 3$. We take $M$ as in step (a). We twice get $h^{1}\left(\mathcal{I}_{S \backslash S \cap M}\left(d_{1}, 0, d_{2}, \ldots, d_{k}\right)\right)>0$. If $\#(S \backslash S \cap M)$ $=2$ we get that $\# \pi(S \backslash S \cap M)=1$ for all $i \neq 1$. Since $S \backslash S \cap M$ is contained in a solution of $q$ and $d_{2}=1$, contradicting the obvious extension $\left(d_{j}\right.$ arbitrary for $\left.j \neq 2\right)$ of $\left[4\right.$, Remark 1.10]. Thus $r_{X}(q)=3$ and hence $\mathcal{S}(X, q) \supseteq\{\{o\} \cup A\}_{A \in \mathcal{S}\left(X^{\prime}, q\right)}$ and in particular $\operatorname{dim} \mathcal{S}(X, q) \geq 1$.

In the same way we get the following result.

Proposition 5.8 Fix $\left(d_{1}, d_{2}\right) \in\{(2,1),(1,2)\}$. Fix an integer $k \geq 3$ and take positive integers $d_{3}, \ldots, d_{k}$. Fix $n_{1}, n_{2} \in\{1$, 
$2\}$ and set $n_{i}:=1$ for all $i=3, \ldots, k$. Fix lines $L_{i} \subseteq \mathbb{P}^{n_{1}}, i=1,2$, and take $o_{i} \in \mathbb{P}^{n_{1}}$; if $n_{i}=2$ assume $o_{2} \notin L$. Fix $e_{i}, o_{i} \in \mathbb{P}^{1}, i=3$, $\ldots, k$ such that $e_{i} \neq o_{i}$ for all $i$. Set $Y:=\mathbb{P}^{n_{1}} \times \mathbb{P}^{n_{2}} \times\left(\mathbb{P}^{1}\right)^{k-2}, Y^{\prime}:=L_{1} \times L_{2} \times\left\{e_{3}\right\} \times \cdots \times\left\{e_{k}\right\} \subset Y, o:=\left(o_{1}, \ldots, o_{k}\right), X:=v_{\left(d_{1}, \ldots, d_{k}\right)}(Y)$ and $X^{\prime}:=v_{\left(d_{1}, \ldots, d_{k}\right)}\left(Y^{\prime}\right)$. Fix $q^{\prime} \in\left\langle X^{\prime}\right\rangle \backslash X^{\prime}$ and take $q \in\left\langle\left\{q^{\prime}, v_{\left(d_{1}, \ldots, d_{k}\right)}(o)\right\}\right\rangle \backslash\left\langle\left\{q^{\prime}, v_{\left(d_{1}, \ldots, d_{k}\right)}(o)\right\}\right\rangle$. Then

(1) $Y$ (resp. $Y^{\prime}$ ) is the minimal multiprojective space containing $q$ (resp. $q^{\prime}$ ).

(2) $r_{X}\left(q^{\prime}\right)=r_{X}\left(q^{\prime}\right)=2, \mathcal{S}\left(X, q^{\prime}\right)=\mathcal{S}\left(X^{\prime}, q^{\prime}\right)$ and $2 \leq r_{X}(q) \leq 3$.

(3) $2 \leq r_{X}(q) \leq 3$.

(4) Assume either $d_{3} \geq 2$ or $k \geq 4$. Then $r_{X}(q)=3$ and $\operatorname{dim} \mathcal{S}(X, q)>0$.

\section{References}

[1] E. Ballico, A. Bernardi. Decomposition of homogeneous polynomials with low rank. Math. Z. 2012; 271: 1141-1149.

[2] E. Ballico, A. Bernardi. Stratification of the fourth secant variety of Veronese variety via the symmetric rank. $A d v$. Pure Appl. Math. 2013; 4(2): 215-250.

[3] E. Ballico, A. Bernardi, M. Christandl, F. Gesmundo. On the partially symmetric rank of tensor products of W-states and other symmetric tensors. Rend. Lincei Mat. Appl. 2019; 30: 93-124.

[4] E. Ballico, A. Bernardi, P. Santarsiero. Identifiability of rank-3 tensors. 2001. p.10497.

[5] K. Baur, J. Draisma. Secant dimensions of low-dimensional homogeneous varieties. Adv. Geom. 2010; 10(1): 1-29.

[6] M. V. Catalisano, A. V. Geramita, A. Gimigliano. Higher secant varieties of Segre-Veronese varieties. In: Projective Varieties with Unexpected Properties. Berlin: Walter de Gruyter; 2005. p.81-107.

[7] M. V. Catalisano, A. V. Geramita A.V., A. Gimigliano. Segre-Veronese embeddings of $\mathbb{P}^{1} \times \mathbb{P}^{1} \times \mathbb{P}^{1}$ and their secant varieties. Collect. Math. 2007; 58(1): 1-24.

[8] C. Ciliberto, M. Mella, F. Russo. Varieties with one apparent double point. J. Algebraic. Geometry. 2004; 13: 475512.

[9] G. Comas, M. Seiguer. On the rank of a binary form. Found. Comp. Math. 2011; 11(1): 65-78.

[10] W. L. Edge. The number of apparent double points of certain loci. Proc. Cambridge Philos. Soc. 1932; 28: 285-299.

[11] R. Hartshorne. Algebraic Geometry. New York: Springer Verlag, Berlin: Heidelberg; 1977.

[12] A. Iarrobino, V. Kanev. Power sums, Gorenstein algebras, and determinantal loci, Lecture Notes in Mathematics. Berlin: Springer-Verlag; 1999. p.1721.

[13] J. M. Landsberg. Tensors: Geometry and Applications, Graduate Studies in Mathematics. Amer. Math. Soc. 2012; 128.

[14] A. Laface. On linear systems of curves on rational scrolls. Geom. Dedicata. 2002; 90: 127144.

[15] A. Laface, E. Postinghel. Secant varieties of Segre-Veronese embeddings of $\left(\mathbb{P}^{1}\right)^{r}$. Math. Ann. 2013; 356: 1455-1470.

[16] A. Laface, L. Ugaglia. Standard classes on the blow-up of $\mathbb{P}^{n}$ at points in very general position. Comm. Algebra. 2012; 40(6): 2115-2129.

[17] F. Russo. On a theorem of Severi. Math. Ann. 2000; 316: 1-17. 